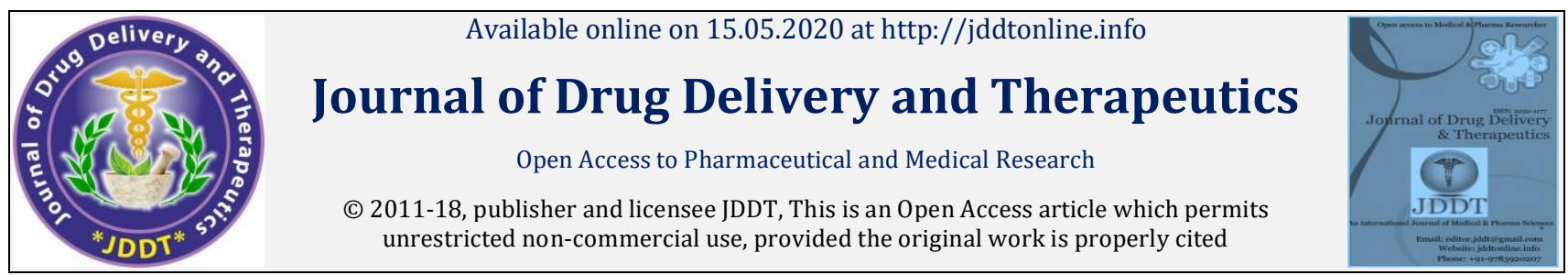

Open $\odot$ Access

Research Article

\title{
A Prospective Observational Study on Rational Use of Antibiotics for Paediatric Patients with Respiratory Tract Infections in a Tertiary Care Hospital
}

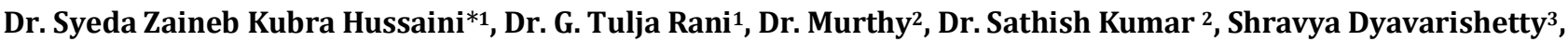 \\ Cherala Laxmi Prasanna ${ }^{3}$, Goli Praneeth ${ }^{3}$
}

${ }^{1}$ Department of Pharmacy Practice, Malla Reddy Pharmacy College, Hyderabad, Telangana, India

2 Department of Paediatrics, Malla Reddy Hospital, Hyderabad, Telangana, India

${ }^{3}$ Pharm D. (Doctor of Pharmacy), Malla Reddy Pharmacy College, Hyderabad, Telangana, India

\begin{abstract}
Background: Antibiotic drug overuse and inappropriate antibiotic drug selection are associated with increased drug resistance among respiratory pathogens (most notably, Streptococcus pneumoniae), possible progression to chronic disease, and increased treatment costs:[1] The selection of an antibiotic for prophylaxis should be based on known or likely target pathogens, for a short duration of time.

Objective: To evaluate Rational use of antibiotics in Paediatric patients suffering from Respiratory tract infections.

Method: It was Prospective, observational study conducted by random selection of patients. Based on diagnosis, the study population was categorized into 2 groups: Upper respiratory tract infections and Lower respiratory tract infections. The study population was interviewed after obtaining written informed consent for information like demographics, diagnosis, treatment and antibiotics prescribed.

Results and Discussion: In our study population, it was observed that male patient (74\%) were higher than female patients (26\%). Total number of antibiotics prescribed during the study period is 83. Most commonly prescribed antibiotic was Augmentin (Amoxicillin + Clavulanic acid) of dose (450mg IV BD) which is the combination drug, i.e, more effective than individual antibiotic drugs.

Conclusion: In the present study it is observed that 4-different classes of antibiotics-Pencillins,Cephalosporins,Macrolides and Aminoglycosides were prescribed and used for different Respiratory diseases.The percentage of average antibiotic utilization is highest in Lower Respiratory Tract Infection with (55.22\%).Mostly used Antibiotic in males for both LRTI and URTI is Augmentin with the percentage of $(52 \%)$ and $(80 \%)$ respectively. Where as in females it is observed that in both LRTI and URTI mostly used Antibiotic is Augmentin with percentage of (38.8\%) and( $80 \%)$ respectively.Overall Second mostly used antibiotic is Ceftriaxone.
\end{abstract}

Keywords: pediatrics, lower and upper respiratory tract infections, antibiotics.

Article Info: Received 04 March 2020; Review Completed 11 April 2020; Accepted 18 April 2020; Available online 15 May 2020

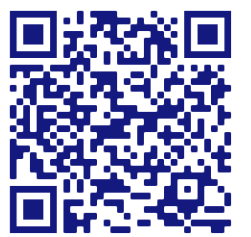

Cite this article as:

Hussaini SZK, Rani GT, Murthy, Kumar S, Dyavarishetty S, Prasanna CL, Praneeth G, A Prospective Observational Study on Rational Use of Antibiotics for Paediatric Patients with Respiratory Tract Infections in a Tertiary Care Hospital, Journal of Drug Delivery and Therapeutics. 2020; 10(3):36-41 http://dx.doi.org/10.22270/jddt.v10i3.4051

Dr. Syeda Zaineb Kubra Hussaini, Department of Pharmacy Practice, Malla Reddy Pharmacy College, Hyderabad, Telangana, India

\section{INTRODUCTION}

Respiratory tract infection refers to any of a number of infectious diseases involving the respiratory tract. Upper respiratory tract includes Nasal cavity, pharynx, Larynx. Lower respiratory tract includes Trachea, Bronchi, bronchioles and lungs. Antibiotics are commonly prescribed for RTI in adults and children in primary care.

Upper respiratory tract infections: Cold and flu, Tonsillitis, Pharyngitis, Otitis media, Influenza, Acute sinusitis.Lower respiratory tract infections: Tracheitis, Laryngitis, Epiglottitis, Bronchiolitis, Pneumonia.[2,3]

\section{Rational Use of Antibiotics}

The rational use of medicines is defined as "patients receive medications appropriate to their clinical needs, in doses that meet their requirements, for an adequate period of time, and at the lowest cost to them and their country".

\section{Guidelines:}

- Evaluate the infection by clinical diagnosis: The clinician should always consider whether or not antibiotic therapy is even necessary for the patient by weighing the benefits (efficacy, rapid recovery and comfort of patient) 
against the risks (antibiotic resistance, adverse effects) and costs of treatment.

- Select an appropriate antibiotic therapy: A clinician should decide whether to direct a patient to a definitive therapy or an empirical therapy or a prophylactic therapy

\section{* Definitive therapy:}

- When the etiology of the infection is known, the clinician should proceed with definitive therapy.

- Empiric the clinician should confirm the bacterial infection

- A negative microbiology test report can make it easier for the clinician to refuse unnecessary prescription of antibiotic.

\section{* Empirical therapy:}

- Clinician should reserve empirical therapy for critical patients, where time is inadequate for identification and isolation of the infection causing bacteria

- $\quad$ prescribing is based on the clinicians working knowledge or experience of what is most likely to be the pathogen causing the patient's condition

* Prophylactic therapy: Antibiotic prophylaxis should be prescribed to susceptible patients to prevent specific infections that can cause definite detrimental effects.

-The selection of an antibiotic for prophylaxis should be based on known or likely target pathogens, for a short duration of time.

Criteria for choosing an antibiotic drug: Antibiotic selection is vital to facilitate successful treatment of infections and minimize the development of antibiotic resistance.

\section{Efficacy:}

1. Narrow spectrum or broad spectrum

2. Monotherapy or combination therapy

3. To achieve synergistic effect

4. Combinational therapy also shortens the course of antibiotic therapy

5. ILL patients require empirical therapy before bacteriological diagnosis

6. To extend antibiotic spectrum during polymicrobial infections

7. To prevent the development of bacterial resistance with long term therapy

8. Efficacy at the site of infection \& tissue penetration

9. Bactericidal vs bacteriostatic therapy

Dosage, Route of Administration and Duration:

1. The clinician should consider pharmacokinetic and pharmacodynamic factors in determining the drug dose

2. Oral/ enteral route of administration should be preferred in patients with mild-to-moderate infections

Monitoring Response to Therapy:

1. The need for an antimicrobial therapy should be reviewed on a daily basis by reviewing laboratory evidence

2. Response to therapy depends on the nature and sensitivity of the agent, specificity of the drug, bioavailability and dosage
3. Longer the doubling time of the organism, longer the time it takes to respond.[4,5]

\section{Antibiotic Resistance}

It is the situation which occurs when the microorganism is resistant to an antibiotic spectrum. These situation is due to changes in the genetic material of the micro oraganism, mutations in one (or)some of the gene (or)with a new gene, by contamination of the organism.

Factors Causing Resistance:

1. Unneccesary antibiotic use

2. Inproper use of antibiotics

3. Incorrrect dosage intervals.

\section{Vaccines}

A vaccine is a biological preparation that provides active acquired immunity to a particular disease. A vaccine typically contains an agent that resembles a disease-causing microorganism and is often made from weakened or killed forms of the microbe, its toxins, or one of its surface proteins.

Vaccines exit for the common influenza viruses ,measles, pertussis, the potentially invasive bacteria haemophilus influenza typeB and many serotypes of streptococcus pneumonia

Influenza Vaccine:

1) Inactivated /Trivalent vaccine-H1N1, H3N2, InfluenzaB

2) Quadrivalent vaccine-H1N1, H3N2 and two strains of influenza B

-As the season usually lasts between the months of OctMay,it is best to get the flu vaccine two week before these period as the body requires time to produce the necessary antibodies to fight the viruses.

Vaccination schedule:-These vaccine can be administered after 6 months of age,2-4 weeks prior to the flu season:(2 doses at the interval of 1 month in the $1^{\text {st }}$ year,and 1 dose annually before the flu season upto 5 years of age.

Pertussis Vaccine: It protects against whooping cough.

There are 2 types:

1) Whole cell vaccine (78\% effective)

2) Acellular vaccines (71-85\% effective)

Vaccination schedule:-It has 3 primary doses at 6,10 and 14 weeks and 2 boosters at 15-18 months and 5 years

Pneumococcal Vaccine: It acts against the streptococcus pneumonia it can prevent pneumonia, sepsis and meningitis

There are 2 types:

1) Conjugate vaccine

2) Polysaccharide vaccine

Vaccination schedule:-_3 doses at 6,10 and 14 weeks with a booster at 15 months

Measles Vaccine: These vaccines prevent measles, that usually their effectiveness last for many years.

They are:

1) Standalone vaccine

2) MR Vaccine 

3) MMR Vaccine
4) MMRV Vaccine

Vaccination schedule:-It is given under Universal Immunization Programme at 9-12 months of age and $2^{\text {nd }}$ dose at 16-24 months of age.

Haemophilus Influenza Type B Vaccine:-It is used to prevent haemophilus influenza type $B$ infection. It is used to decrease in the rate of meningitis, pneumonia and epiglottitis.

Vaccination schedule:-It was given at 2,4,6 months and as a booster dose at 12-15 months

\section{Objectives:}

- To evaluate inappropriate antibiotic drug selection those are associated with increased drug resistance among respiratory pathogens.

- Detailed information on commonly prescribed antibiotics for RTI.

- To evaluate the problem of the increase in antibiotic resistance.

\section{METHODS AND METHODOLOGY:}

Inclusion Criteria Includes:

- $\quad$ Children in P.I.C.U

- Children in paediatric department suffering from respiratory tract infections

- Children whose legal guardians are willing to give informed consent for the study

- Children of age below 12 years are included.
- Both genders are included.

Exclusion Criteria:

- Adults are excluded from the study

- Geriatrics are excluded

- Pregnant and lactating women are excluded.

\section{RESULTS}

Patient Population Demographics

Table 1: Distribution of patients based on Gender

\begin{tabular}{|c|c|c|}
\hline Sex & Patients & Percentage \\
\hline Male & 40 & 74 \\
\hline Female & 20 & 26 \\
\hline Total & $\mathbf{6 0}$ & $\mathbf{1 0 0}$ \\
\hline
\end{tabular}

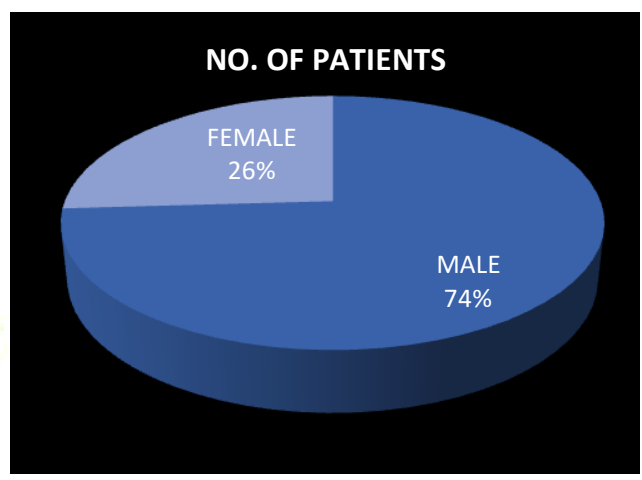

Figure 1: Distribution of patients based on Gender

Table 2: Distribution of patients based on Age groups

\begin{tabular}{|ccccccc|}
\hline AGE(Yrs $)$ & $0-3$ & $4-7$ & $8-11$ & \multicolumn{1}{c}{$12-15$} & $16-18$ \\
\hline MALE & 26 & 5 & 7 & 1 & 0 \\
\hline
\end{tabular}

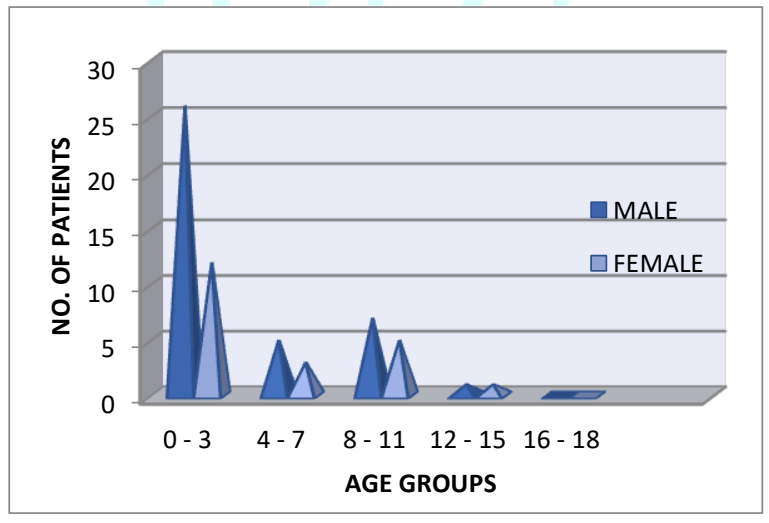

Figure 2: Distribution of patients based on Age groups

Table 3: Distribution of patients based on type of infection

\begin{tabular}{|c|c|c|c|} 
Age Group & \multicolumn{2}{|c}{ Sex } & \multicolumn{2}{c}{} \\
\cline { 3 - 4 } & & LRTI & URTI \\
\hline Pediatrics & Male & 31 & 8 \\
\cline { 2 - 4 } & Female & 16 & 5 \\
\hline & Total & 47 & 13 \\
\hline & Percentage & $78.3 \%$ & $21.66 \%$ \\
\hline
\end{tabular}




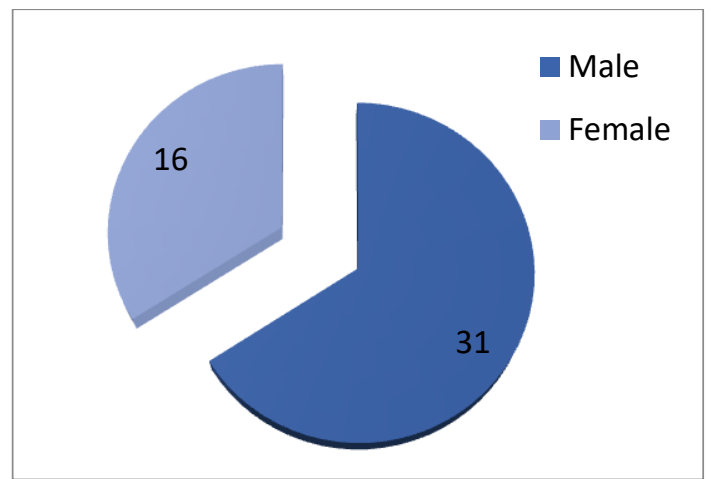

Figure 3.1: Distribution of patients with LRTI

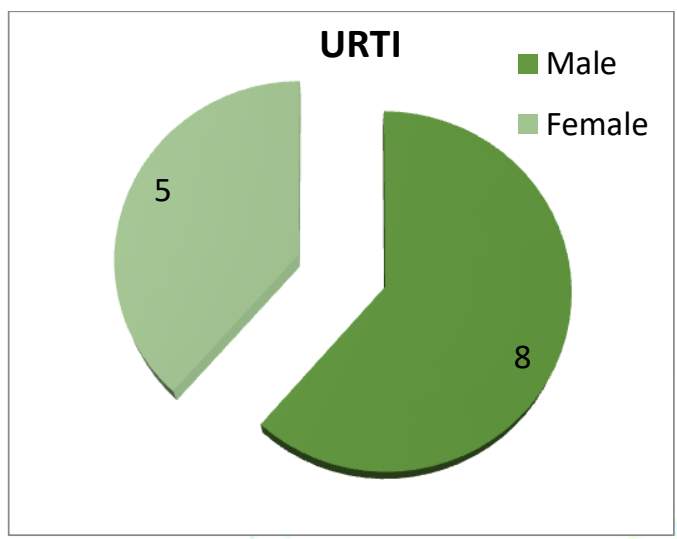

Figure 3.2: Distribution of patients with URTI

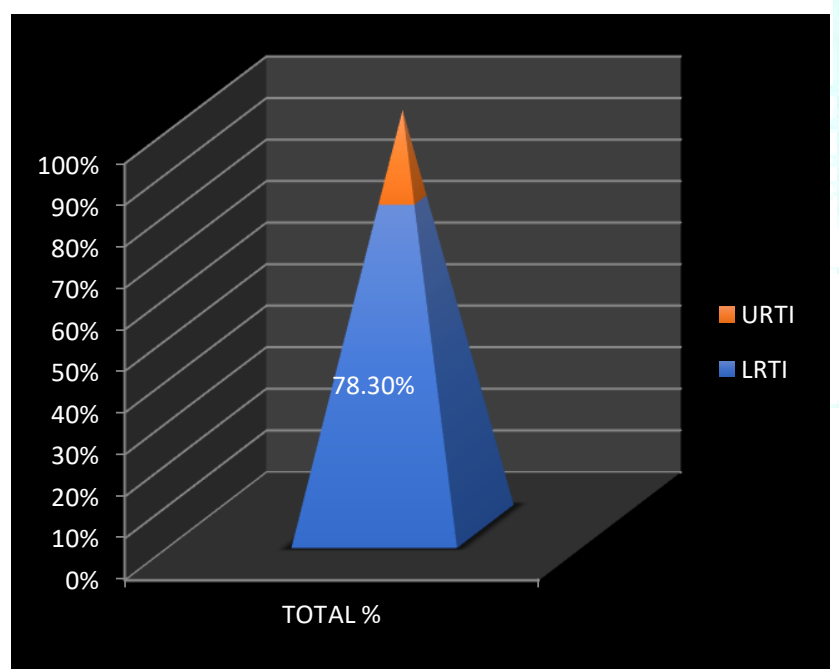

Figure 3.3: Percentage of patients based on type of infection

Table 4: Distribution of underlying co-morbidities in patients

\begin{tabular}{|l|c|}
\hline CO-MORBID DISEASES & NO. OF PATIENTS \\
\hline Endocrine disorders & 1 \\
\hline Seizures & 1 \\
\hline Anemia & 13 \\
\hline Down syndrome & 1 \\
\hline None & 44 \\
\hline
\end{tabular}

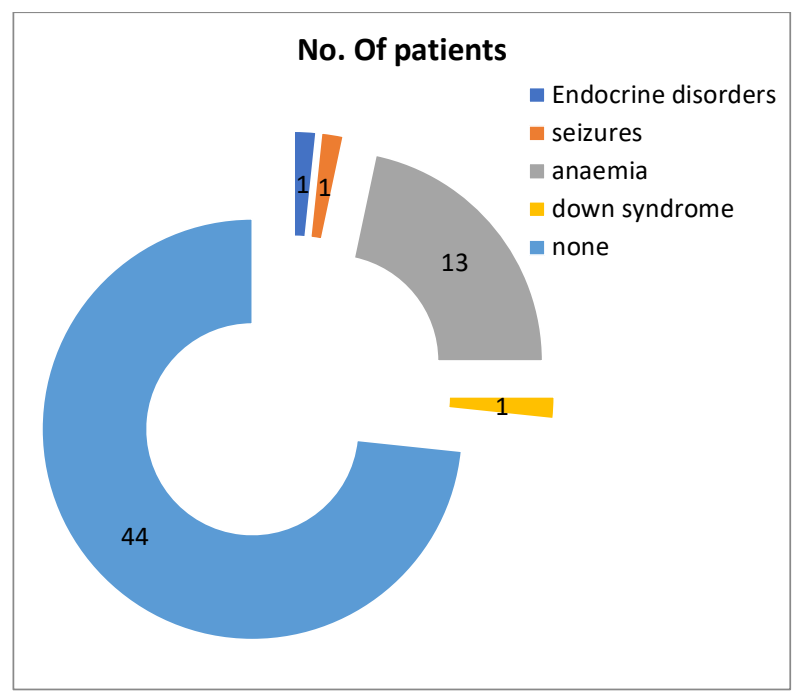

Figure.4: Distribution of underlying co-morbidities in patients

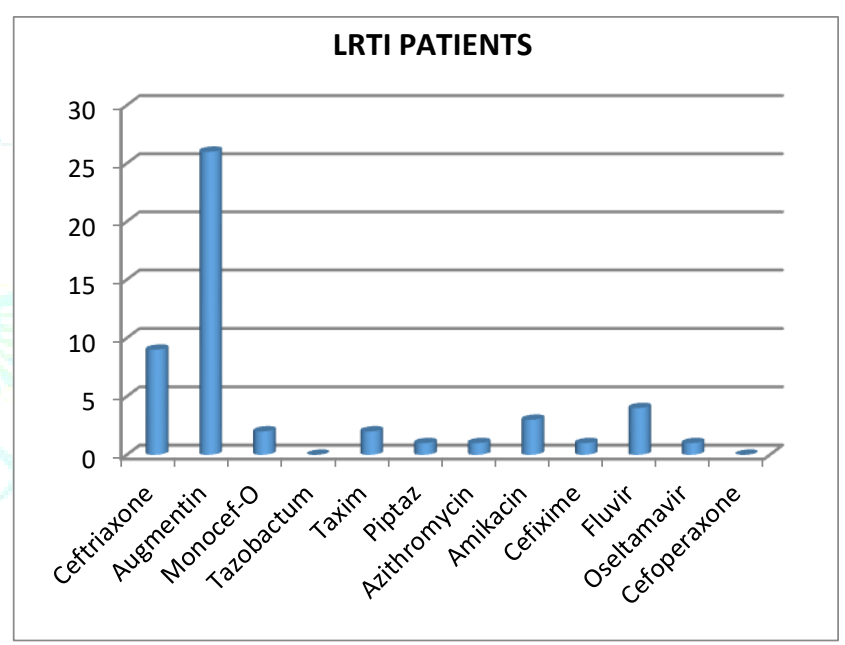

Figure 5.1: Drugs prescribed in Males with LRTI

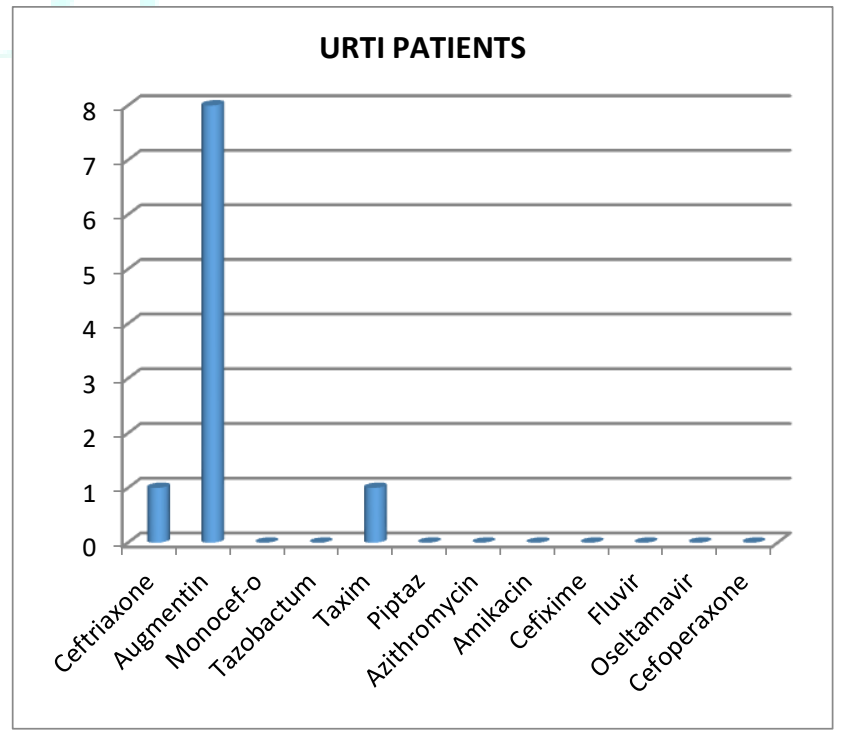

Figure 5.2: Drugs prescribed in Males with URTI 


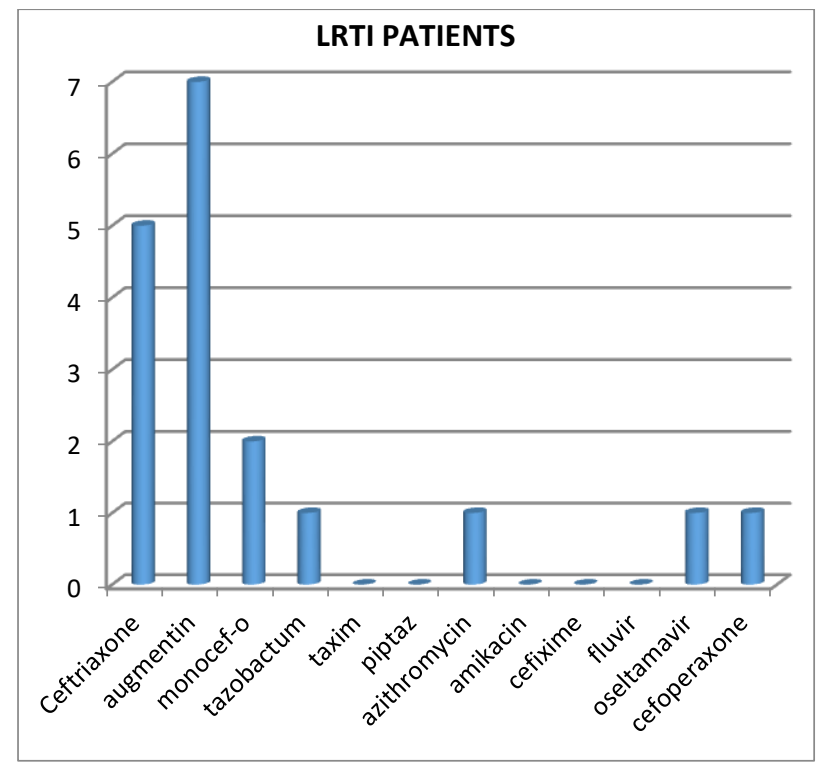

Figure 5.3: Drugs prescribed in females with LRTI

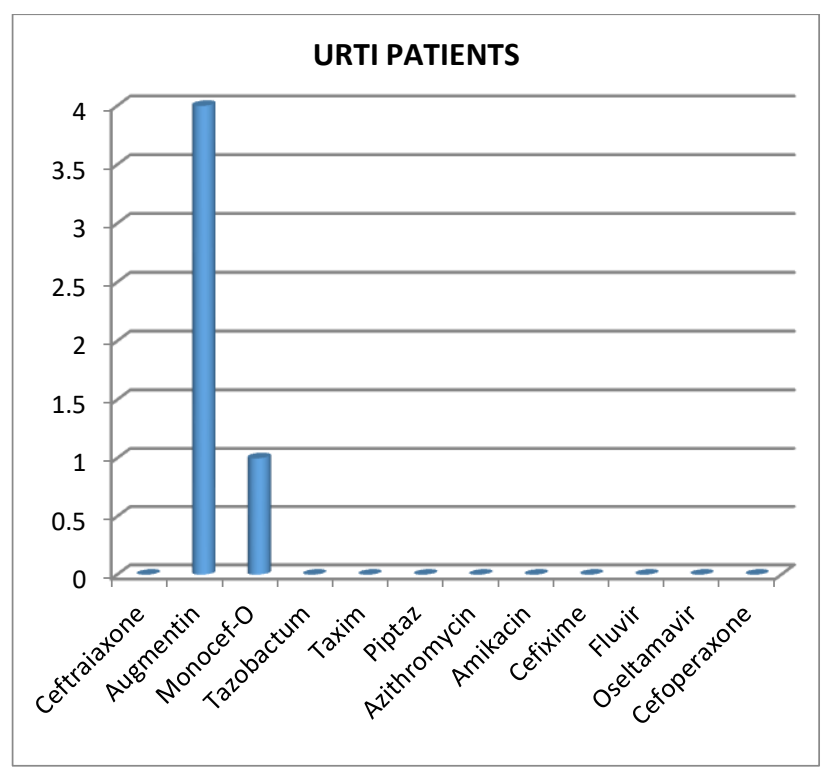

Figure 5.4: Drugs prescribed in Females with URTI

Table 5: Percentage utilization of different classes of Antibiotics in patients

\begin{tabular}{|c|c|c|c|c|}
\hline Antibiotics & LRTI & URTI & TOTAL & \% Percentage \\
\hline Penicillin & 33 & 4 & 37 & 55.22 \\
\hline Cephalosporin & 23 & 2 & 25 & 37.31 \\
\hline Macrolide & 2 & 0 & 2 & 2.98 \\
\hline Aminoglycoside & 3 & 0 & 3 & 4.47 \\
\hline Total & 61 & 6 & 67 & 100 \\
\hline
\end{tabular}

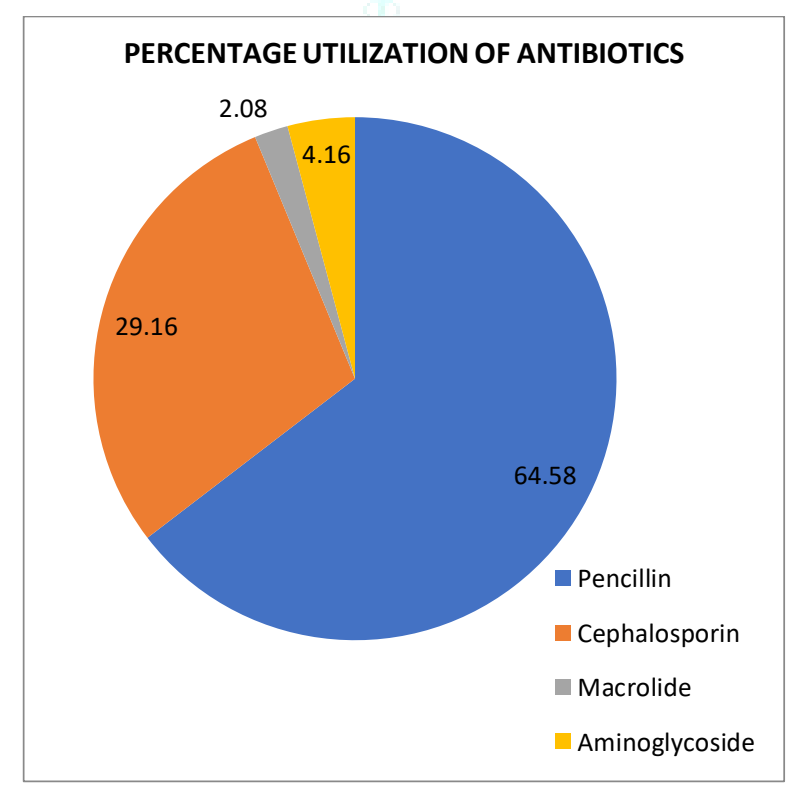

Figure 6: Percentage utilization of different classes of Antibiotics in patients 


\section{CONCLUSION}

RTI in children are one of the most common reasons for parents consulting health professionals.Most Respiratory tract infections are self limiting viral illnesses that will resolve with time and supportive management,but coming to bacterial infections there is an need for rational use of antibiotics.However,it is important for health professional to identify any RTI that may have more serious implications,for the child and require medical intention .[6,7]

\section{Sample population demographics:}

In this "A Prospective observational study on Rational use of antibiotics for paediatric in RTI,"were evaluated in a total of 60 patients.Among 60 patients,(74\%) are males and (26\%) are females.The total study populations were categorized in to 5 groups,depending up there age groups i.e,(0-3),(4-7),(811),(12-15),(16-18) years.From the above categorized age groups ,age of (0-3) years mostly in males are infected,with Lower Respiratory Tract Infection.From the collected data it is known that most of the RTI patients are with Anaemia.The risk of Acute respiratory tract infection was due to iron deficiency anemia,as in which adequate iron is important for maturation of immune cells ,mainly lymphocytes .These lymphocytes helps in generation of specific response to infection.In these present study, it is observed that patients with wheeze (or) crepts sounds,along cough with sputum have Shortness of breathe.

\section{Antibiotics utilization in Respiratory infections:}

In the present study it is observed that 4-different classes of antibiotics-Pencillins-[Augmentin, Tazobactum, Piptaz], Cephalosporins -[Ceftriaxone, Monocef-0, Taxim, Cefixime, Cefoperaxone], Macrolides-[Azithromycin] and Aminoglycosides -[Amikacin] were prescribed and used for different Respiratory diseases.The percentage of average antibiotic utilization is highest in Lower Respiratory Tract Infection with (55.22\%).Mostly used Antibiotic in males for both LRTI and URTI is Augmentin with the percentage of $(52 \%)$ and $(80 \%)$ respectively.Where as in females it is observed that in both LRTI and URTI mostly used Antibiotic is Augmentin with percentage of (38.8\%) and( $80 \%)$ respectively.Overall Second mostly used antibiotic is Ceftriaxone.

Treatment with antibiotics is neccessary for certain diseases,inorder to ensure survival,welfare,productivity, and to reduce spread of diseases:[10,11]The goal of rational use initiatives is not always to reduce antibiotic use,but instead ensure that the use is appropriate.Rational prescribing and rational drug use are important goals to achieve appropriate treatment to patients.[12,13] From the above prescribed drugs ,some of the drugs are $1^{\text {st }}$ line drugs that include Ceftriaxone(350mg), Augmentin(450mg), Monocef-O(50mg), Taxim(90mg), Amikacin(120mg) and Cefixime(2.5ml syrup).
These drugs are considered as Rational Drugs i.e, appropriate medicines are prescribed in the correct dose and dosage form to the correct patient and at the lowest cost to the patient and the community.[8,9]. Due to the irrational use of drugs, there is an development of resistance to antibiotics, ineffective treatment, adverse effects, drug dependence which is an major problem in current medical practice. So,there is an urgent need to use mainly rational drugs to promote appropriate treatment to the children.[14]

\section{REFERENCES}

1. Thomas M. File, Jr, MD; and James A. Hadley, MD Rational Use of Antibiotics to Treat Respiratory Tract Infections, The American journal of managed care, 8(8):713.

2. WHO EMRO | Volume 23, 2017

3. Shankar P, Upadhay D, Subish B. Prescribing patterns among paediatric inpatients teaching hospital in Western Nepal. Singapore Med J. 2006; 47(4):261-5

4. Andrews T, Thompson M, Buckley DI, Heneghan C, Deyo R, Redmond $\mathrm{N}$, et al. Interventions to influence consulting and antibiotic use for acute respiratory tract infections in children: a systematic review and meta-analysis. PLoS ONE 2012; 7(1):e30334. [DOI: 10.1371/journal.pone.0030334]

5. Davey P, Marwick CA, Scott CL, Charani E, McNeil K, Brown E, et al. Interventions to improve antibiotic prescribing practices for hospital inpatients. Cochrane Database of Systematic Reviews 2017, Issue 2. Art. No.: CD003543 DOI:

6. Pakhale, Smita; Mulpuru, Sunita; Verheij, Theo J. M.; Kochen, Michael M.; Rohde, Gernot G. U.; Bjerre, Lise M. (2014-10-09)" description about respiratory infections.

7. Pramod kerkar MD, FFARCSI, Pain assist. Cast modified on:March27,2017.https://www.epainassist.com/face-mouththroat/what-is-upper-respiratory-tract-infection.

8. Bellet P.S. Upper respiratory tract infection (the common cold), in: R.C. Baker (Ed.), Pediatric primary care III - child care. Lippincott, Williams \& Wilkins, Philadelphia, PA, 2001, pp. $1-384$.

9. World Health Organization. The rational use of drugs. World Health Organization, Geneva, 1987.

10. Schroeder K., Fahey T. Over the counter medications for acute cough in children and adults in ambulatory settings. Cochrane Database Syst. Rev. (2001) CD001831. Comment in: Evid. Based Nurs. 2002; 5(3):78.

11. Fahey T., Stocks N., Thomas T. Systematic review of the treatment of upper respiratory tract infection. Arch. Dis. Child 1998; 79:225- 230.

12. Jhaj R., Bhargava V.K., Uppal R., Lekha S., Reeta K., Kaur N. Use of cold medications for upper respiratory tract infections in children. Pharmacoepidemiol. Drug Saf. 2001; 10:323- 327.

13. Ching-Lan Cheng, Yea-Huei Kao Yang, Ching-Chuan Liu and Ping-Ing Lee, A retrospective study on the usage of cough and cold medications in viral respiratory tract infections in Taiwanese children, Pharmacoepidemiology and Drug Safety, 2013; 23(1):36-42).

14. Biswadeep Das, Chayna Sarkar and Abhijeet Guha Majumder, Medication use for pediatric upper respiratory tract infections, Fundamental \& Clinical Pharmacology, 2006; 20(4):385-390. 